\title{
LA POTENCIACIÓN DE LA COMPETENCIA TRADUCTOLÓGICA EN ALUMNOS UNIVERSITARIOS: UNA PROPUESTA HÍBRIDA
}

\author{
PROMOTING TRANSLATION COMPETENCE IN \\ UNIVERSITY STUDENTS: A HYBRID PROPOSAL
}

\author{
Nicolás Montalbán Martínez \\ Universidad de Murcia \\ nicolas.montalban@um.es
}

Fecha de recepción: 17-09-2019

Fecha de aceptación: 22-12-2019

\section{RESUMEN}

El objetivo del presente artículo es comprobar la potenciación de la competencia traductológica por medio de un acercamiento incluyendo aprendizaje combinado, aula invertida, Aprendizaje Basado en Proyectos y gamificación. Para ello, se empleará la plataforma Sakai en una experiencia didáctica con los alumnos de las asignaturas Traducción para el Turismo y Ocio I (Inglés), Traducción para el Turismo y Ocio II (Inglés) y Traducción Especializada (Inglés), del tercer y cuarto curso del grado de Traducción e Interpretación, en el curso académico 2018/2019 en la Universidad de Murcia. Para evaluar dicha experiencia se realizó en primer lugar una evaluación inicial para comprobar el estado de partida de los alumnos participantes en la experiencia. En el transcurso de la intervención didáctica, los alumnos desarrollaron actividades fuera de clase empleando la herramienta Foro del Aula Virtual (utilizada para completar actividades y organizar un debate continuo), Tareas (para ubicar los encargos de traducción), etc., conjuntamente con actividades en clase como exposiciones, mini-debates, evaluaciones empleando Kahoots y prácticas de traducción. Al 
final de todo el proceso se evaluaron las competencias traductológicas, y se envió a los alumnos una encuesta final. Se concibe este estudio como un proyecto piloto para evaluar la viabilidad de los instrumentos y herramientas utilizadas, así como su diseño, previo a su implantación a mayor escala en otras asignaturas.

Palabras clave: Aprendizaje combinado; Aula invertida; Aprendizaje Basado en Proyectos (ABP); Aprendizaje basado en juegos.

\section{ABSTRACT}

The aim of this article is to find out if translation competence could be promoted by introducing a hybrid approach including Blended Learning, Flipped classroom, Project-based learning and Gamification. To do that, the Sakai platform is going to be introduced in a didactic experience with students of the subjects Translation for Tourism and Leisure I (English), Translation for Tourism and Leisure II (English) and Specialized Translation (English), belonging to the third and fourth year of the university degree Translation and Interpreting in 2018/2019 at the University of Murcia. To evaluate the aforementioned experience, an initial evaluation was implemented to know the starting point of the students of the subjects. In the didactic intervention, students implemented activities out of the classroom using the Discussion Forum (to develop activities and organize a continuous debate), Tasks (to upload the translations), etc., together with activities in class, such as oral presentations, mini-debates, evaluations using Kahoots and translation practices. At the end of the process the translation competences were evaluated and students completed a final questionnaire. This is a pilot project to assess the viability of used tools, as well as its design, prior to its implementation on a larger scale in other subjects.

Key words: Blended Learning; Flipped classroom; Project-based learning; Game-based learning

\section{INTRODUCCIÓN}

Por aprendizaje combinado («blended learning») se entiende un acercamiento pedagógico híbrido definido por una serie de procesos variados y prácticos. Generalmente cuando se considera el término aprendizaje combinado nos estamos refiriendo al lugar donde se produce el aprendizaje, la combinación del aula y el entorno «online» (Graham, 2013). Esta forma de trabajo ha sido ampliamente implantada en los entornos de enseñanza universitaria, siendo denominado como «nuevo modelo tradicional» (Ross y Gage 2006: 167), o «normalidad actual» (Norberg, Dziuban y Moskal 2011: 207). La introducción del aprendizaje combinado nos lleva a plantearnos las señas de identidad de la sociedad digital y del uso de las tecnologías de la información y comunicación (TIC). La mayoría de autores hablan de una sensación cada vez mayor de que vivimos en una sociedad dominada por la información (Floridi, 2008). 
Para Graham (2013) el aprendizaje combinado es la integración de la enseñanza presencial (o sincrónica) con la desarrollada en línea (asincrónica). Hay autores que se han centrado en su potencial efectividad (Garrison y Kanuka 2004; Picciano 2009). La investigación llevada a cabo en torno al tema ha reforzado la idea de la mejora del aprendizaje de los alumnos (Dziuban y Moskal 2011; Dziuban, Hartman, Cavanagh, y Moskal, 2011; Means, Toyama, Murphy, y Baki, 2013), además de un sentido comunitario evidente (Rovai y Jordan 2004). Conviene reseñar cinco meta análisis sobre el impacto del aprendizaje combinado en relación a su efectividad (Zhao, Yan, Lai, y Tan, 2005; Sitzmann, Kraiger, Stewart, y Wisher, 2006; Bernard, Abrami, Borokhovski, Wade, Tamim, Surkes, y Bethel 2009; Means, Toyama, Murphy, Kaia, y Jones 2010; Bernard, Borokhovski, Schmid, Tamim, y Abrami 2014), obteniendo resultados moderadamente a favor del aprendizaje combinado cuando se compara con la enseñanza estrictamente presencial.

Margulieux, McCracken y Catrambone (2016: 105-111) definen los cursos de aprendizaje combinado con las siguientes categorías:

—Localización: el lugar específico donde se desarrolla.

- Medio de distribución.

— Modalidad de instrucción: magistral, activo, etc.

— Sincronía: actividades simultáneas, sucesivas en la línea de tiempo.

Hay autores que han estudiado el gran potencial del aprendizaje combinado en el desarrollo de competencias transversales. Willem, Aiello y Bartolomé (2007) han observado la competencia digital en los entornos del aprendizaje combinado, influyendo notablemente en la intención y motivación por aprender (Zhang, Dang y Amer, 2016). El desarrollo de la docencia en estos entornos también favorece el denominado aprendizaje autorregulado, siguiendo las consideraciones de Whiteside, Dikkers y Lewis (2016). Otros autores han centrado sus estudios en el aumento del trabajo colaborativo implícito al aprendizaje combinado (Fernández-Sánchez, 2011).

La introducción de esta metodología de aprendizaje supone el empleo de nuevos elementos y herramientas tecnológicas (Sife, Lwoga, y Sanga, 2007). En esta intervención didáctica se han introducido:

—Un sistema de gestión del aprendizaje.

- Algunas herramientas de comunicación virtual, como los correos electrónicos y los foros.

— Los documentos y manuales para descargarse.

- El aula invertida

— Los trabajos colaborativos por proyectos.

En el presente trabajo los alumnos y el profesor han empleado la plataforma de aprendizaje en línea denominada Sakai, la cual dispone de un Aula Virtual, que ofrece acceso directo a profesores y alumnos a distintas herramientas que favorecen la comunicación por medio de mensajería, anuncios, foros y chat, además de posibilitar la inclusión de los materiales docentes, y el desarrollo de tareas complementarias. Esta es la herramienta que 
pueden emplear los docentes y alumnos de la Universidad de Murcia. Otras posibilidades que ofrece la la plataforma son las ventanas de Recursos (donde se pueden ubicar materiales didácticos de diverso formato), Foro, Mensajes Privados, Chat, etc. Las características más importantes de Sakai (Sakai Learning Management System; Sakai 11.3 release) son:

—Está diseñado para favorecer el aprendizaje colaborativo.

- Es capaz de ofrecer distintas herramientas para la comunicación.

- Posee una arquitectura abierta, con la posibilidad de integrar herramientas de otras arquitecturas.

— Tiene el estándar IMS («Learning Tools Interoperability»).

- Dispone de una alta flexibilidad a la hora de crear y diseñar el curso, y elegir las herramientas necesarias.

Para Ouadoud, Yassin y Nejjari (2018: 132-161) Sakai tiene una gran riqueza pedagógica y funcional basada en el constructivismo y es extremadamente modular. En el estudio comparativo de estos autores con otras plataformas, Sakai obtuvo un $94 \%$ en usabilidad y seguridad, $92 \%$ en idoneidad funcional, $90 \%$ en sostenibilidad, así como un $80 \%$ en portabilidad, situándose en segundo lugar después de Moodle.

En esta experiencia híbrida se ha introducido el aula invertida. Para Olaizola (2014: 1) «se podría clasificar la «clase invertida» como una forma específica de blended- learning, es decir, un modelo que integra educación presencial y educación a distancia». Walvoord y Johnson Anderson (1998), propusieron un modelo de aula invertida en el que los alumnos, antes de ir a clase, tienen un primer acercamiento con los contenidos, y es en el aula donde se favorece la comprensión del contenido (sintetizando, analizando y resolviendo problemas). Lage, Platt y Treglia (2000) aplicaron una metodología de aula invertida en un curso universitario de Introducción a la Economía. Estos autores prepararon una serie de materiales didácticos con actividades que trasladaban la instrucción directa fuera del aula. El tiempo de clase se empleaba en actividades expositivas, debates y discusiones en los que se analizaba y aplicaba principios económicos. Crouch y Mazur (2001) plantearon un modelo en el que el alumno trabajaba el material antes de ir a clase, la cual se dedicaba a mini-lecciones y preguntas conceptuales sobre los materiales. Bergmann y Sams (2012) conjuntamente con Fornons y Palau (2016), aplicaron también el Aula Invertida, obteniendo una mejora sustancial en la participación del alumnado y en su actitud, además de favorecer un ambiente de trabajo positivo. Asimismo, Marlowe (2012) realizó una experiencia didáctica con alumnos universitarios, y advirtió una disminución importante del nivel de estrés en los alumnos. Por otro lado, Flumerfelt y Green (2013) aplicaron esta técnica en alumnos de secundaria con dificultades, observándose un aumento importante de los procesos participativos «online» y del trabajo fuera del ámbito del aula. Roach (2014) y Elliot (2014) obtuvieron también resultados positivos en la aplicación de esta metodología, observando un aumento de las actividades realizadas fuera del aula. Es necesario reseñar los estudios de O’Flaherty y Phillips (2015), que realizaron una propuesta de múltiples actividades síncronas y asíncronas. Los resultados de esta experiencia abundan en la gran motivación que desarrollan los alumnos, además de aumentar la eficiencia del aprendizaje. Cieliebak y Frei (2016) y Jonsson (2015) procedieron a evaluar los resultados sobre competencias no 
técnicas en alumnos de ingeniería, empleando el Aula Invertida, observando mejores resultados si se comparan con aquellos obtenidos empleando métodos tradicionales. Para Perdomo (2016), la aplicación del aula invertida potencia la comunicación de forma asertiva, el aprendizaje significativo y la motivación de los alumnos.

El trabajo colaborativo por proyectos es uno de los recursos mencionados para el aprendizaje combinado. En este modelo los alumnos planifican, llevan a cabo y evalúan proyectos aplicables a distintos proyectos más allá del aula (Blank, 1997, Dickinson, et al., 1998, Harwell, 1997). En la actualidad estamos asistiendo al desarrollo de metodologías docentes centradas en el aprendizaje del estudiante y que muestran una mayor implicación del alumnado en el proceso de enseñanza (Vega et al., 2014). Nos referimos a las metodologías activas y entre ellas al Aprendizaje Basado en Proyectos (ABP). En esta metodología, el alumno se convierte en el principal protagonista de todo el proceso educativo y el profesor en un mediador del mismo (Vizcarro y Juárez, 2008). Es de reseñar que esta metodología ha sido muy utilizada en la formación de docentes universitarios (Benítez et al., 2013). El ABP fomenta a los alumnos a aprender a aprender, además de trabajar de forma colaborativa en grupo, buscando soluciones a un problema planteado por el docente. Es, además, una ayuda extraordinaria en la adquisición del aprendizaje autodirigido (Saverv, 2006), pudiendo trabajar de forma autónoma y alcanzando mejores resultados (Thomas et al., 1999). El ABP puede ser una herramienta fundamental para el desarrollo de competencias como trabajar de forma colaborativa, el empleo de redes sociales, la motivación, la creatividad y la solución de problemas. Los alumnos que trabajan en entornos ABP expresan una mayor capacidad resolutiva de problemas (Finkelstein et al., 2010).

\subsection{Uso de Kahoot}

En la presente experiencia didáctica se ha empleado la plataforma Kahoot, que es una herramienta de gamificación con un software gratis. Fue diseñada por Johan Brand, Jamie Brooker y Morten Versvik, y desarrollada en la Universidad de Ciencia y Tecnología de Noruega. Para registrarse en la plataforma hay que acceder a la siguiente dirección: https:// create.kahoot.it/login. Una vez registrado, se puede acceder a un menú en el que se encuentran unas instrucciones muy simples para cualquier alumno o profesor, un apartado en el que se pueden crear nuevos kahoots partiendo de los ya existentes o de nueva creación, y otra sección en la que se puede acceder a los distintos informes de los resultados de las distintas pruebas planteadas. El formato del juego Kahoot es el de una serie de preguntas con opción múltiple a las que se pueden agregar vídeos e imágenes para atraer más la atención de los alumnos. Hay varias modalidades de test como el «Quiz, Jumble, Discussion y Survey». Cuando se activa la opción de jugar, inmediatamente se crea un código pin que los jugadores han de introducir en sus dispositivos (ordenadores, tabletas y teléfonos móviles), y los juegos se muestran en una pantalla compartida. Al estar los jugadores presentes en el aula se estimula la discusión grupal. Para el profesor, el uso de esta herramienta sirve para evaluar y recoger la información de los alumnos. Mientras los alumnos aprenden jugando, 
se les puede someter a desafíos, repasar conocimientos, o simplemente permitir que se diviertan creando grupo. Es de destacar que es posible utilizar cualquier dispositivo electrónico para la plataforma Kahoot (Plump y LaRosa, 2017). Este software gratuito facilita la creación de cuestionarios en relación a un tema específico, favoreciendo la aplicación práctica de los conocimientos (Caraballo, Peinado y González, 2017), de tal forma que es capaz de producir un ambiente de actividad invitando a los alumnos a participar e involucrarse en los resultados (Moreno, Ureña, Luna, De la Cruz, Roldán, Castro, Aguagliaro, Salmerón, Gil, y Alcaide, 2018). Esta experiencia de gamificación deviene en una competencia motivadora e ilusionante tanto individual como grupal (Jiménez, Gámez y Gómez, 2016; Ramos y Botella, 2017). Los resultados del juego se pueden observar en un archivo en Excel generado por el propio software, en el que se pueden ver las respuestas acertadas y el tiempo empleado en responder, creando un «ranking».

Con el presente estudio se pretende averiguar si la combinación de todas las metodologías mencionadas potencia la adquisición de la competencia traductora. La competencia traductora ha sido tradicionalmente definida atendiendo al modelo de competencia, es decir, aquellas habilidades, destrezas y actitudes necesarias para desarrollar una actividad de forma satisfactoria, incidiendo fundamentalmente en la formación y el trabajo del traductor (Király 1995: 108). Neubert (2000: 3-18) es de la opinión de que la competencia traductora integra una serie de competencias que incluyen, entre otras, la competencia en la lengua origen y meta. Además de ello, este autor distingue cinco competencias que definen la competencia traductora: la competencia en la lengua, la textual, específica, cultural y de transferencia. El grupo de investigación PACTE (2003) propuso un Modelo de Competencia Traductora basado en cinco subcompetencias y componentes psico-fisiológicos (2005: 610-611). La primera subcompetencia es la bilingüe (Hurtado Albir, 2015: 256-280, la cual consiste en el sistema subyacente de conocimientos y destrezas necesarias para la la comunicación lingüística, e incluye la competencia gramatical, la textual, la ilocucionaria (relacionada con las funciones del lenguaje) y la sociolingüística. A continuación, tenemos la subcompetencia extralingüística, conformada por el conocimiento bicultural y temático. Después está la subcompetencia asociada al conocimiento de la traducción (procesos, métodos y procedimientos), la subcompetencia instrumental (uso de fuentes de documentación y tecnologías de la información) y la subcompetencia estratégica, que integra todas las mencionadas.

\section{METODOLOGÍA}

\subsection{Contextualización}

La experiencia didáctica se implementó a los diecinueve alumnos universitarios de la asignatura Traducción para el Turismo y el Ocio I (Inglés), a los veinticinco alumnos de la asignatura Traducción para el Turismo y el Ocio II (Inglés) y a los diecinueve alumnos de la asignatura Traducción Especializada (Inglés), del tercer y cuarto curso del grado de Traducción e Interpretación, cursos todos ellos desarrollados en 2018/2109. 


\subsection{Diseño y desarrollo de la experiencia didáctica}

El diseño y desarrollo de la experiencia se estructura en torno a las siguientes fases:

—-Diagnóstico inicial de las competencias traductológicas.

- Desarrollo de la asignatura.

—Evaluación final de las competencias traductológicas y de la experiencia didáctica.

\subsection{Diagnóstico inicial de las competencias traductológicas}

Para averiguar los conocimientos iniciales del alumnado en torno a sus conocimientos previos de la materia, se procedió a realizar un diagnóstico inicial consistente en una serie de preguntas relacionadas con los contenidos a desarrollar. Estas preguntas se formularon el primer día de clase, tras finalizar la presentación de la asignaturas. Para ello se empleó una herramienta denominada Slido, en la que se creó una encuesta con distintas preguntas relativas a la presentación. La asignatura Traducción para el Turismo y Ocio I incluyó las siguientes preguntas: ¿Conoces los géneros textuales del turismo y sus características?, ¿Qué recursos y estrategias conoces para la traducción de textos turísticos?, ¿Distingues tipos de textos promocionales de viajes y sus características? y ¿Qué recursos web hay para la traducción de textos turísticos?. También es importante reseñar las preguntas de la asignatura Traducción para el Turismo y Ocio II: ¿Sabes que es un foro y un blog de viaje?, ¿Has leído algún libro de viaje? ¿Puedes identificar las características de los libros de viaje?, ¿Conoces tipos de textos promocionales de viajes y sus características?, ¿Cómo se comprueba la calidad de los textos turísticos? y ¿Has trabajo con herramientas de traducción automática?. En cuanto a las cuestiones de la asignatura Traducción Especializada son las siguientes: ¿Sabes qué tipo de errores se presentan en la traducción de un texto especializado?, ¿Puedes identificar los principales problemas al traducir textos turísticos y de publicidad?, ¿Conoces los principales problemas de la traducción de textos socioeconómicos, jurídicos y administrativos? y Enumera los problemas de la traducción científico-técnica.

\subsection{Desarrollo de la asignatura}

En la realización de este estudio se utilizaron varios tipos de materiales con los que estructurar una secuencia de tareas que permitiera cuantificar la competencia de los alumnos en relación a los contenidos. Estos materiales didácticos se ubicaron en el Aula Virtual en la carpeta Recursos de las distintas asignaturas, separados por unidades:

- Artículos científicos.

— Una serie de vídeos tutoriales.

— Enlaces a páginas web.

— Prácticas de traducción de textos tratados.

Los textos para las prácticas de la asignatura Traducción para el Turismo y el Ocio I (Inglés) son, fundamentalmente, textos turísticos atendiendo al género, guías turísticas, 
folletos, textos publicitarios y webs turísticas. En cuanto a la asignatura Traducción para el Turismo y el Ocio II (Inglés) hay que considerar textos provenientes de foros y blogs de viaje, fragmentos de libros de viajes, textos promocionales del turismo, así como textos académicos y divulgativos. En la tercera asignatura Traducción Especializada (Inglés), hay textos orientados al consumo, socioeconómicos y científico-técnicos.

En la carpeta Foro, se organizó una discusión permanente en torno a los contenidos fundamentales de la asignatura, con preguntas que suscitaron el debate entre los alumnos.

También se emplearon las carpetas Anuncios, con las fechas de entrega de trabajos, Tareas (para entregar las prácticas evaluables) y Mensajes Privados. Para desarrollar todos los temas se realizaron actividades asincrónicas y sincrónicas. Dentro de las actividades asincrónicas que se prepararon en grupo cabe distinguir entre teóricas y prácticas. Para el desarrollo de las actividades teóricas el profesor realizó una serie de preguntas de cada tema en el Foro y los alumnos, divididos en grupos, respondieron a las preguntas. Los materiales de consulta de cada Tema estaban ubicados en Recursos y consistían en vídeos explicativos, hipervínculos a páginas en internet y documentos en PDF (artículos de consulta). Todos los alumnos (al tener acceso a todos los materiales a través del aula virtual) visionaron y estudiaron los vídeos, leyeron los artículos, etc., con el objetivo de comprender los contenidos. Cada uno de los Temas se asignó a un grupo de alumnos para que, ya en el aula, realizara una exposición sobre los aspectos teóricos en forma de debate moderado por el profesor. Tras la exposición de cada grupo, el grupo asignado procedió a responder todas las dudas. Una vez concluida la exposición de cada tema, se valoraron los conocimientos aprendidos por todos los alumnos a través de un cuestionario Kahoot confeccionado por el grupo encargado de la exposición. Este cuestionario fue revisado, antes de su exposición en clase, por parte del profesor, para comprobar que cubría todos los objetivos y puntos principales de cada tema. Para las prácticas de traducción, se procede de forma análoga. Aunque todos los alumnos realizaron todas las prácticas de traducción distribuidos en grupos, se le asignó la exposición razonada de las traducciones en el aula a distintos grupos, seguida de un debate moderado por el profesor en torno a dudas planteadas. Las prácticas de traducción van conformando un proyecto final, que es la elaboración de un Blog profesional elaborado por los propios alumnos, constituyendo todo un producto final de la asignatura. 


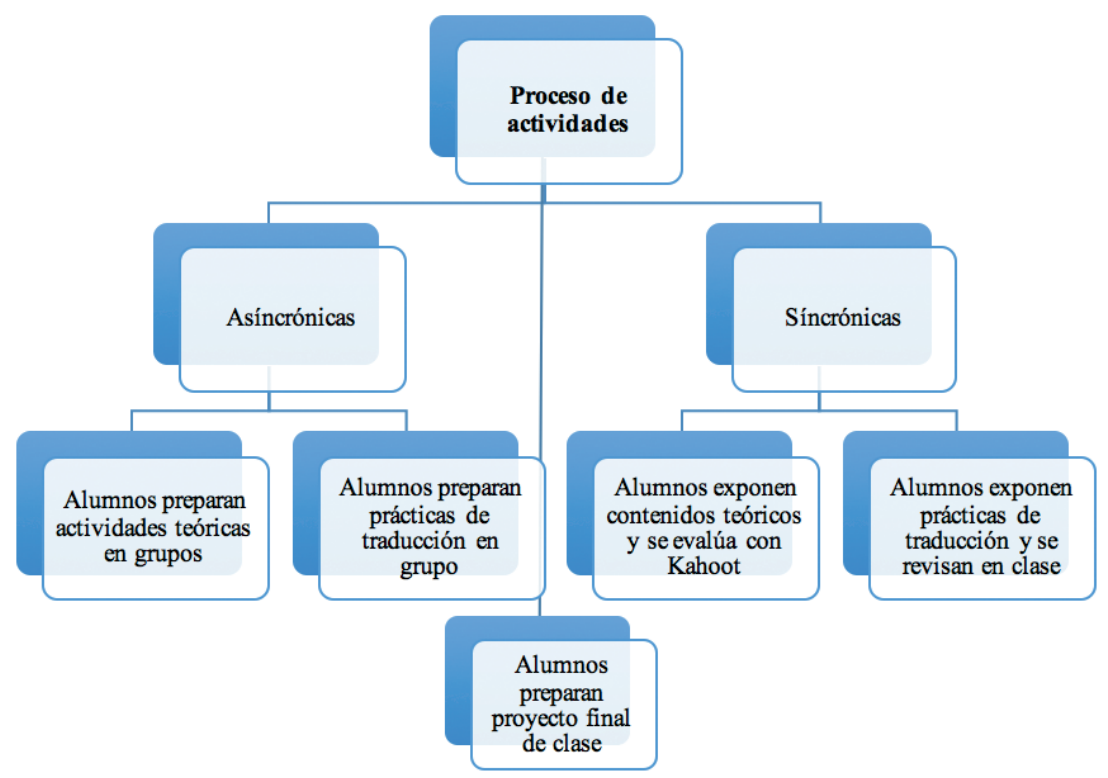

FIGURA I.

Esquema del proceso de actividades

Para los Kahoots confeccionados por los alumnos sobre cuestiones teóricas se aplicó la siguiente rúbrica:

TABLA I.

Rúbrica de evaluación de los Kahoots elaborados por los alumnos

\begin{tabular}{|l|l|}
\hline Métodos / Instrumentos & $\begin{array}{l}\text { - Evaluación por medio de un ejercicio } \\
\text { de kahoot de los Temas teóricos. }\end{array}$ \\
\hline Criterios de Valoración & $\begin{array}{l}\text { Asimilación de los contenidos teóricos y } \\
\text { aproximación de las traducciones de los } \\
\text { alumnos a las de referencia. }\end{array}$ \\
\hline Ponderación & $10 \%$ \\
\hline
\end{tabular}

Para estudiar la calidad de las prácticas de traducción se compararon las traducciones de los alumnos con las de referencia por medio de una serie de métricas de evaluación. Las primeras métricas que se introdujeron fueron la Precisión y la Exhaustividad. En primer lugar se computó el número de palabras de ambas traducciones (la de los alumnos y las de referencia). Para calcular la Precisión el número de palabras comunes se dividió por el número de palabras de la traducción de referencia. Se considera que las traducciones son buenas si los resultados son altos, de tal forma las mejores traducciones son las que tienen los 
resultados mas altos. Otra métrica que se utilizó es el «WER (Word Error Rate)» o índice de Error de las Palabras. Este método considera diferencias tales como sustituciones, inserciones o supresiones. Esta métrica está basada en la distancia de Levenshtein que se calcula al nivel de la palabra. En este caso, cuanto menor es el índice mejor es el resultado. Pero la métrica mas usada es el «BLEU (Bilingual Evaluation Understudy)». Este método calcula cuantos n-gramas se solapan entre las traducciones de los alumnos y las de referencia. Esta métrica está basada sobre la hipótesis de que cuanto mayor sea el número de n-gramas que se solapen entre las traducciones de los alumnos y las de referencia, mejores son las traducciones realizadas. La fórmula que calcula «BLEU» es la siguiente:

$$
\mathrm{BLEU}=\min \left(1, \frac{\text { number of words in } \mathrm{MT}}{\text { number of words in ref }}\right) \prod_{i=1}^{4} \text { precision }_{i}
$$

Para obtener los resultados de las métricas de evaluación se desarrolló un programa informático ${ }^{1}$, escrito en lenguaje Python, que calcula «WER», «BLEU», «Precision» $\mathrm{y}$ «Recall» a partir de la información volcada en un archivo. El archivo reconoció un encabezamiento, seguido de diferentes segmentos de texto que se correspondían con el original, la traducción de los alumnos y la de referencia, para proceder a su comparación. El código calculó cada función combinando las traducciones de los alumnos con las de referencia, generando otro archivo con formato de tabla que se envió directamente a una hoja de cálculo. El programa calculó la media teniendo en cuenta las métricas mencionadas. Aplicando la siguiente fórmula utilizada para la rúbrica de las prácticas de traducción:

$(3 *(1-\mathrm{W})+1 * \mathrm{~B}+1 * \mathrm{P}+1 * \mathrm{R}) \cdot 10 / 6$

si $\mathrm{W}=0$ no hay errores, siendo la máxima puntuación $1-\mathrm{W}$

se calculó una puntuación aproximada para las traducciones de los textos.

TABLA 2.

Rúbrica de evaluación de las prácticas de traducciones

\begin{tabular}{|l|l|}
\hline Métodos / Instrumentos & $\begin{array}{l}\text { - Evaluación por medio de un programa } \\
\text { informático que calcula métricas de } \\
\text { evaluación y puntuación aproximada: } \\
(3 *(1-\mathrm{W})+1 * \mathrm{~B}+1 * \mathrm{P}+1 * \mathrm{R}) \cdot 10 / 6\end{array}$ \\
\hline Criterios de Valoración & $\begin{array}{l}\text { Comparación de las traducciones de los } \\
\text { alumnos con las de referencia. }\end{array}$ \\
\hline Ponderación & $30 \%$ \\
\hline
\end{tabular}

1 Este programa fue desarrollado por Juan Manuel Dato Ruiz (técnico informático cualificado), teniendo en cuenta las métricas de evaluación explicadas anteriormente. 


\subsection{Evaluación final de las competencias traductológicas y de la experiencia didáctica}

Para la evaluación final de las nuevas competencias traductológicas adquiridas el profesor empleó un Kahoot para la parte teórica con preguntas de todos los temas tratados y una traducción para la parte práctica.

TABLA 3.

Rúbrica de evaluación del Kahoot de la parte final teórica

\begin{tabular}{|l|l|}
\hline Métodos / Instrumentos & $\begin{array}{l}\text { - Evaluación por medio de un ejercicio } \\
\text { de kahoot de los Temas teóricos. }\end{array}$ \\
\hline Criterios de Valoración & $\begin{array}{l}\text { Asimilación de los contenidos teóricos y } \\
\text { aproximación de las traducciones de los } \\
\text { alumnos a las de referencia. }\end{array}$ \\
\hline Ponderación & $10 \%$ \\
\hline
\end{tabular}

TABLA 4.

Rúbrica de evaluación de la traducción del examen final basada en Hurtado Albir (1995: 49-74).

\begin{tabular}{|c|c|c|c|c|c|c|}
\hline \multicolumn{2}{|c|}{ Métodos / Instrumentos } & \multicolumn{5}{|c|}{$\begin{array}{l}\text { Evaluación por medio de un } \\
\text { examen con un texto similar a los } \\
\text { realizados en el aula. }\end{array}$} \\
\hline \multicolumn{7}{|c|}{ Criterios de Valoración } \\
\hline \multicolumn{2}{|c|}{ Nivel de competencia } & 1 & 2 & 3 & 4 & 5 \\
\hline \multicolumn{2}{|c|}{ Puntuación de los elementos ponderables } & $0-2$ & $3-4$ & $5-6$ & $7-8$ & $9-10$ \\
\hline $\begin{array}{l}\text { Comprensión del } \\
\text { texto original }\end{array}$ & $\begin{array}{l}\text { - Contrasentido } \\
\text { - Falso sentido } \\
\text { - Sin sentido } \\
\text { - Adición innecesaria } \\
\text { - Supresión innecesaria } \\
\text { - Alusiones extralingüísticas } \\
\text { no solucionadas } \\
\text { - No mismo sentido } \\
\text { - Inadecuación de variación } \\
\text { lingüística }\end{array}$ & & & & & \\
\hline
\end{tabular}




\begin{tabular}{|c|c|c|c|c|c|c|}
\hline \multicolumn{2}{|c|}{ Métodos / Instrumentos } & \multicolumn{5}{|c|}{$\begin{array}{l}\text { Evaluación por medio de un } \\
\text { examen con un texto similar a los } \\
\text { realizados en el aula. }\end{array}$} \\
\hline \multicolumn{7}{|c|}{ Criterios de Valoración } \\
\hline \multicolumn{2}{|c|}{ Nivel de competencia } & 1 & 2 & 3 & 4 & 5 \\
\hline \multicolumn{2}{|c|}{ Puntuación de los elementos ponderables } & $0-2$ & $3-4$ & $5-6$ & $7-8$ & $9-10$ \\
\hline $\begin{array}{l}\text { Expresión en la } \\
\text { lengua de llegada }\end{array}$ & $\begin{array}{l}\text { - Ortografía y puntuación } \\
\text { - Gramática } \\
\text { - Léxico } \\
\text { - Textual } \\
\text { - Estilística }\end{array}$ & & & & & \\
\hline $\begin{array}{l}\text { Inadecuación fun- } \\
\text { cional }\end{array}$ & $\begin{array}{l}\text { - A la función textual priorita- } \\
\text { ria del original } \\
\text { - A la función de la traducción }\end{array}$ & & & & & \\
\hline \multicolumn{2}{|l|}{ Ponderación } & \multicolumn{5}{|l|}{$50 \%$} \\
\hline
\end{tabular}

En el examen de la parte teórica de la asignatura Traducción para el Turismo y Ocio I, se incluyeron preguntas relativas a las convenciones de los textos turísticos en inglés y en español, sobre los géneros discursivos del turismo, las fuentes documentales para la traducción turística, las guías turísticas, los folletos, textos publicitarios y webs turísticas. En Traducción para el Turismo y Ocio II el examen teórico final consideró preguntas sobre retos y estrategias para la traducción de textos de nivel avanzado, aspectos relacionados con foros, blogs y libros de viaje, las características de diferentes textos promocionales tanto visuales como escritos y los principales retos de un texto turístico académico y divulgativo. En la última de las asignaturas, Traducción Especializada, el examen teórico final valoró los conocimientos de los alumnos sobre cuestiones como la traducción de los nombres propios, lugares de interés turístico, culturemas, cartas comerciales e INCOTERMS y sobre los textos científico-técnicos. Al finalizar la asignatura, los alumnos valoraron la experiencia didáctica por medio de una encuesta diseñada con la herramienta Google Forms. Dicha encuesta midió una serie de parámetros con la escala psicométrica de Likert, con un rango del 1-5, donde 1 equivalía a nada, y 5 a mucho. Los instrumentos empleados en las distintas fases del experimento (diagnóstico inicial, evaluación intermedia y final para la evaluación de las competencias, así como los resultados), nos servirán de pilotaje para determinar si podemos utilizarlos posteriormente a gran escala en otras asignaturas con las modificaciones pertinentes para mejorarlos.

\section{RESULTADOS}

Centrándonos en los resultados de la evaluación inicial, es necesario referirse a a cada una de las asignaturas. 
En relación a Traducción para el Turismo y Ocio I, los alumnos declararon no conocer los géneros textuales del turismo. En cuanto a los recursos y estrategias para la traducción de estos textos, algunos alumnos mencionaron algunas técnicas (Molina \& Hurtado Albir, 2002) dadas en otras asignaturas del grado de Traducción e Interpretación. Cabe destacar la adaptación, el calco, la descripción, el préstamo y la traducción literal, pero sin ofrecer ejemplos concretos. En cuanto a los recursos web, un alumno mencionó IATE (Inter Active Terminology for Europe). En cuanto a las traducciones, algunos alumnos presentaban los siguientes problemas: omisiones no justificables, gramática, ortografía, terminología inapropiada, falsos sentidos y contrasentidos.

En cuanto a la asignatura Traducción para el Turismo y Ocio II, todos los alumnos manifestaron conocer qué es un foro, un blog de viaje y habían leído algún libro de viaje y textos promocionales de turismo, pero no conocían todas sus características. En cuanto a la traducción turística avanzada, todos los textos presentaron algún problema en la traducción, como por ejemplo omisiones no justificables, errores de gramática, ortografía, puntuación, orden de las palabras, terminología inapropiada, falsos sentidos, etc. No obstante, analizadas las traducciones de forma global y teniendo en cuenta que todos los alumnos provienen de la asignatura Traducción para el Turismo y el Ocio I (Inglés) (desarrollada en el cuatrimestre anterior), puede decirse que los alumnos tienen competencia traductológica en este ámbito a nivel intermedio.

Refiriéndonos a Traducción Especializada, todos los alumnos se refirieron a la tipología de problemas de traducción (Hurtado Albir, 2001) dada en casi todas las asignaturas del grado. En este sentido, los alumnos mencionaron problemas de tipo cultural, temático, así como aquellos derivados del encargo de traducción, las características del destinatario y el contexto en que se realiza la traducción. No se aportó ningún ejemplo concreto. Si nos referimos a las traducciones realizadas en clase, podemos observar una disminución de los problemas que aparecen anteriormente, y algunos introducen omisiones no justificables, errores gramaticales, puntuación y ortografía, terminología inapropiada, falsos sentidos, etc.

En el desarrollo de las tres asignaturas, es interesante reseñar que los resultados de los Kahoots realizados tras los debates/exposiciones de cada Tema son positivos, con una media de $97,5 \%$ de respuestas correctas y $2,5 \%$ de respuestas incorrectas.

En cuanto a las prácticas de traducción, se adjunta la media de las métricas de evaluación de todos los alumnos en los todos textos traducidos en las Tablas 1, 2 y 3, así como una puntuación aproximada de los mismos.

TABLA 5 .

Resultados de los alumnos en las traducciones realizadas en Turismo y Ocio I

\begin{tabular}{|l|c|c|c|c|c|c|}
\hline & Alumno & WER & BLEU & PRE & REC & NOTA \\
\hline Turismo I & & & & & & \\
\hline Traducción 1 & 1 & 0,6173 & 0,0684 & 0,9599 & 0,5865 & 4,6 \\
\hline & 2 & 0,7692 & 0,0004 & 0,8575 & 0,6508 & 3,7 \\
\hline
\end{tabular}




\begin{tabular}{|c|c|c|c|c|c|c|}
\hline & Alumno & WER & BLEU & PRE & REC & NOTA \\
\hline \multicolumn{7}{|l|}{ Turismo I } \\
\hline & 3 & 0,7066 & 0,0016 & 0,8435 & 0,6719 & 4 \\
\hline & 4 & 0,723 & 0,0083 & 0,8363 & 0,7727 & 4,1 \\
\hline \multirow[t]{4}{*}{ Traducción 2} & 5 & 0,6667 & 0,2013 & 0,9706 & 0,8996 & 5,1 \\
\hline & 6 & 0,5275 & 0,0771 & 0,9373 & 0,9089 & 5,6 \\
\hline & 7 & 0,4836 & 0,2145 & 0,9696 & 0,8958 & 6 \\
\hline & 8 & 0,6924 & 0,1227 & 0,8893 & 0,8632 & 4,7 \\
\hline \multirow[t]{4}{*}{ Traducción 3} & 9 & 0,5815125 & 0,1592375 & 0,916371875 & 0,835525 & 5,2 \\
\hline & 10 & 0,5252 & 0,201 & 0,9346 & 0,8595 & 5,7 \\
\hline & 11 & 0,4982 & 0,2182 & 0,9372 & 0,9073 & 5,9 \\
\hline & 12 & 0,4303 & 0,3749 & 0,9178 & 0,8706 & 6,5 \\
\hline \multirow[t]{4}{*}{ Traducción 4} & 13 & 0,5978 & 0,1543 & 0,9649 & 0,8936 & 5,4 \\
\hline & 14 & 0,2404 & 0,4046 & 0,9832 & 0,8996 & 7,6 \\
\hline & 15 & 0,6094 & 0,1644 & 0,923 & 0,8748 & 5,2 \\
\hline & 16 & 0,62 & 0,0299 & 0,9073 & 0,8889 & 4,9 \\
\hline \multirow[t]{3}{*}{ Traducción 5} & 17 & 0,4425 & 0,1176 & 0,9623 & 0,9129 & 6,1 \\
\hline & 18 & 0,2883 & 0,3227 & 0,974 & 0,9296 & 7,3 \\
\hline & 19 & 0,4452 & 0,1862 & 0,9315 & 0,8947 & 6,1 \\
\hline
\end{tabular}

TABLA 6.

Resultados de los alumnos en las traducciones realizadas en Turismo y Ocio II

\begin{tabular}{|l|c|c|c|c|c|c|}
\hline & Alumno & WER & BLEU & PRE & REC & NOTA \\
\hline Turismo II & & & & & & \\
\hline Traducción 1 & 1 & 0,3359 & 0,184 & 0,7451 & 0,7999 & 6,2 \\
\hline & 2 & 0,2694 & 0,3253 & 0,826 & 0,8692 & 7 \\
\hline & 3 & 0,3896 & 0,1586 & 0,78 & 0,8399 & 6 \\
\hline
\end{tabular}




\begin{tabular}{|c|c|c|c|c|c|c|}
\hline & Alumno & WER & BLEU & PRE & REC & NOTA \\
\hline \multicolumn{7}{|l|}{ Turismo II } \\
\hline & 4 & 0,1636 & 0,311 & 0,8933 & 0,9606 & 7,8 \\
\hline & 5 & 0,1681 & 0,245 & 0,9036 & 0,955 & 7,7 \\
\hline & 6 & 0,3577 & 0,5189 & 0,961 & 0,9914 & 7,3 \\
\hline \multirow[t]{4}{*}{ Traducción 2} & 7 & 0,0885 & 20210,5213 & 0,9614 & 0,9943 & 8,7 \\
\hline & 8 & 0,0615 & 0,62223237 & 0,9648 & 0,9954 & 9 \\
\hline & 9 & 0,0664 & 0,511245 & 0,9466 & 0,9847 & 8,7 \\
\hline & & & 25 & & & \\
\hline \multirow[t]{5}{*}{ Traducción 3} & 10 & 0,2824 & 0,2162 & 0,9355 & 0,9838 & 7,1 \\
\hline & 11 & 0,78 & 0,6232 & 0,9723 & 0,9809 & 8,9 \\
\hline & 12 & 0,4291 & 0,432 & 0,9255 & 0,9452 & 6,7 \\
\hline & 13 & 0,0298 & 0,901 & 0,9902 & 0,9902 & 9,7 \\
\hline & 14 & 0,331 & 0,4581 & 0,9556 & 0,9678 & 7,3 \\
\hline \multirow[t]{5}{*}{ Traducción 4} & 15 & 0,87 & 0,6191 & 0,968 & 1 & 8,9 \\
\hline & 16 & 0,1487 & 0,3645 & 0,9271 & 0,9753 & 8 \\
\hline & 17 & 0,147 & 0,4994 & 0,9377 & 0,9758 & 8,3 \\
\hline & 18 & 0,222 & 0,5295 & 0,9595 & 0,9857 & 8 \\
\hline & 19 & 0,0537 & 0,6565 & 0,9593 & 0,9751 & 9 \\
\hline \multirow[t]{6}{*}{ Traducción 5} & 20 & 0 & 1 & 1 & 1 & 10 \\
\hline & 21 & 0,0446 & 0,6684 & 0,9594 & 1 & 9,2 \\
\hline & 22 & 0,1183 & 0,7107 & 0,9749 & 0,982 & 8,9 \\
\hline & 23 & 0,0733 & 0,5715 & 0,9571 & 0,9722 & 8,8 \\
\hline & 24 & 0,0995 & 0,5396 & 0,9227 & 0,9676 & 8,6 \\
\hline & 25 & 0,2809 & 0,3836 & 0,8941 & 0,9875 & 7,4 \\
\hline
\end{tabular}


TABLA 7.

Resultados de los alumnos en las traducciones realizadas en Traducción Especializada

\begin{tabular}{|c|c|c|c|c|c|c|}
\hline & Alumno & WER & BLEU & PRE & REC & NOTA \\
\hline \multicolumn{7}{|l|}{$\begin{array}{l}\text { Traducción } \\
\text { especializada }\end{array}$} \\
\hline \multirow[t]{4}{*}{ Traducción 1} & 1 & 0,9354 & 0,0169 & 0,7482 & 0,3107 & 2,1 \\
\hline & 2 & 0,8053 & 0,1579 & 0,493 & 0,4254 & 2,8 \\
\hline & 3 & 0,8363 & 0,0915 & 0,5843 & 0,5077 & 2.8 \\
\hline & 4 & 1 & 0 & 0,1 & 0,25 & 0,6 \\
\hline \multirow[t]{4}{*}{ Traducción 2} & 5 & 0,2 & 0,3125 & 1 & 0,9048 & 7,7 \\
\hline & 6 & 0,6458 & 0,0106 & 0,7911 & 0,7736 & 4,4 \\
\hline & 7 & 0,5247 & 0,041 & 0,9428 & 0,8411 & 5,4 \\
\hline & 8 & 0,6882 & 0,0008 & 0,9106 & 0,6765 & 4,2 \\
\hline \multirow[t]{3}{*}{ Traducción 3} & 9 & 0,5254 & 0,2003 & 0,9445 & 0,8595 & 5,7 \\
\hline & 10 & 0,4982 & 0,2182 & 0,9352 & 0,9073 & 5,9 \\
\hline & 11 & 0,4303 & 0,3749 & 0,9188 & 0,8706 & 6,5 \\
\hline \multirow[t]{3}{*}{ Traducción 4} & 12 & 0,4016 & 0,3757 & 0,934 & 0,9002 & 6,7 \\
\hline & 13 & 0,6936 & 0,0019 & 0,9175 & 0,8419 & 4,5 \\
\hline & 14 & 0,4063 & 0,2126 & 0,9339 & 0,544 & 6,3 \\
\hline \multirow[t]{5}{*}{ Traducción 5} & 15 & 0,518 & 0,0703 & 0,9523 & 0,8244 & 5,5 \\
\hline & 16 & 0,5474 & 0,1757 & 0,9015 & 0,8642 & 5,5 \\
\hline & 17 & 0,2466 & 0,6605 & 0,9361 & 0,9111 & 7,9 \\
\hline & 18 & 0,3488 & 0,0409 & 0,9881 & 0,8408 & 6,4 \\
\hline & 19 & 0,4085 & 0,205 & 0,9649 & 0,8881 & 6,4 \\
\hline
\end{tabular}

Considerando la evaluación final de la parte teórica realizada con un Kahoot que incluye preguntas de la totalidad de los Temas, el resultado es coincidente con la media de los Kahoots realizados al final de cada tema durante el desarrollo de la asignatura $(97,5 \%$ 
de respuestas correctas y $2,5 \%$ de respuestas incorrectas). Asimismo, los resultados de la evaluación final de la parte práctica (traducción de textos especializados) muestran un resultado altamente positivo, obteniendo los diecinueve alumnos de la primera asignatura, los veinticinco alumnos de la segunda y los veinte de la última, un resultado comprendido entre el 9-10.

La encuesta final enviada a los alumnos que evaluó la propuesta didáctica implementada, en torno a la combinación de todas las metodologías mencionadas en la potenciación de la competencia traductológica, incluye preguntas como si las herramientas son atractivas, si son creativas y motivadoras, si son efectivas y útiles para la adquisición de nuevas competencias traductológicas, además de replicables en otras asignaturas. La pregunta asociada a la adquisición de la nueva competencia traductólogica (véase Figura 4) contenía varios apartados relativos a la valoración final de la adquisición de las subcompetencias asociadas: la bilingüe, la extralingüística, la asociada al conocimiento de la traducción, la instrumental y la estratégica. Los siguientes diagramas de sectores muestran los resultados de la encuesta.

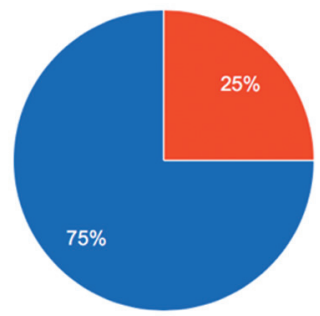

FIGURA 2.

Punto relativo a si son atractivas las herramientas

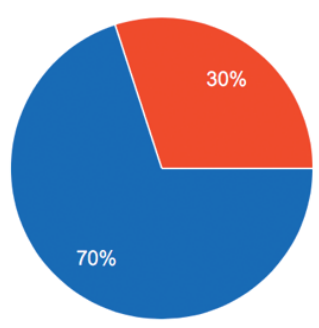

FIGURA 4.

Punto relativo a si los materiales son motivadores
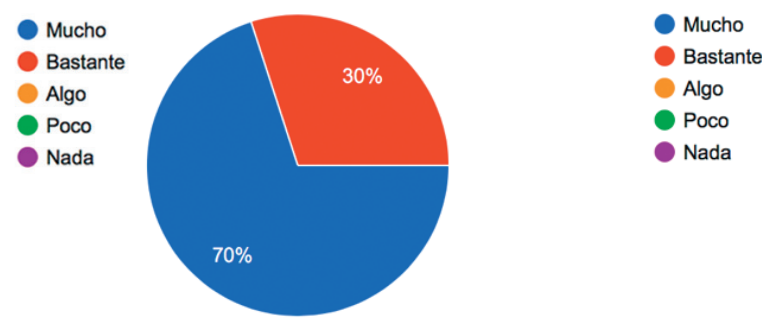

FIGURA 3.

Punto relativo a la creatividad de los materiales
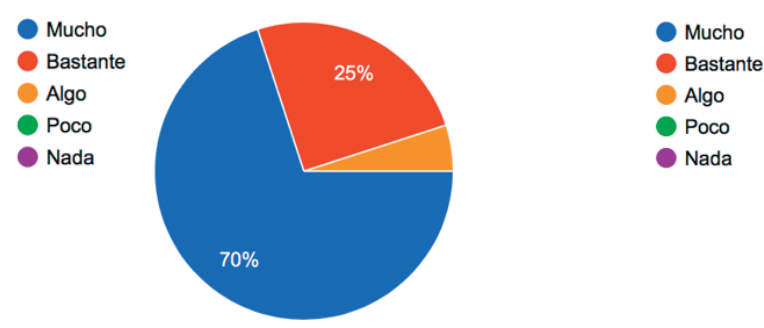

FIGURA 5.

Punto relativo la efectividad y utilidad de los materiales para la adquisición de la nueva competencia 


\section{FIGURA 6.}

Punto relativo a la replicabilidad de la experiencia didáctica en otros contextos y asignaturas

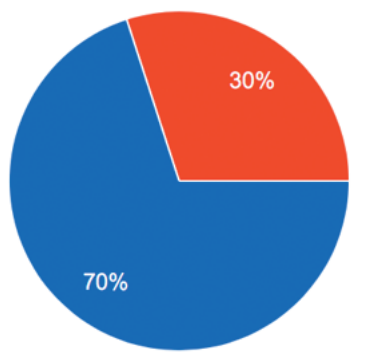

Mucho

Bastante

Algo

Poco

Nada

Como vemos en las Figuras de arriba la mayoría de los alumnos considera que las herramientas empleadas son atractivas, creativas, motivadoras, efectivas y útiles. Además de ello, los alumnos consideran que los materiales les han ayudado para la consecución de la competencia traductológica. También es importante la opinión mayoritaria manifestada en cuanto a la replicabilidad de la experiencia en otras asignaturas y contextos.

\section{DISCUSIÓN}

Si se considera que los alumnos parten de una situación deficitaria en lo que se refiere a los conocimientos teóricos de la nueva asignatura, a tenor de lo expuesto más arriba, es innegable que, tras el desarrollo de la materia y su implementación mediante un ambiente cooperativo de trabajo y focalizado hacia un proyecto final en el entorno de aprendizaje que combina distintas metodologías, los alumnos adquieren un conocimiento avanzado de los mismos, como puede verse tras la realización de los Kahoots en clase (97,5\% de respuestas correctas y 2,5\% de respuestas incorrectas). En cuanto a las prácticas de traducción realizadas grupalmente de forma asincrónica, siendo posteriormente expuestas y debatidas en clase todas las dudas, se aprecia una consolidación progresiva de los siguientes criterios: omisiones no justificables, gramática, ortografía, puntuación, orden de palabras, terminología inapropiada, incoherencia, inconsistencias, falsos sentidos, contrasentidos, problemas en la transmisión del lenguaje, aplicación de estrategias de traducción y resolución de problemas. El estudio de las métricas de evaluación (WER, BLEU, Precision, Recall y nota media aproximada de cada alumno en las cinco traducciones) muestra claramente una progresiva mejora en cuanto a la adquisición de la competencia traductora se refiere. En las tres figuras que aparecen a continuación se aprecia el progreso de las notas aproximadas en las cinco traducciones. 
FigURA 7.

Notas aproximadas de las traducciones en la asignatura Turismo I

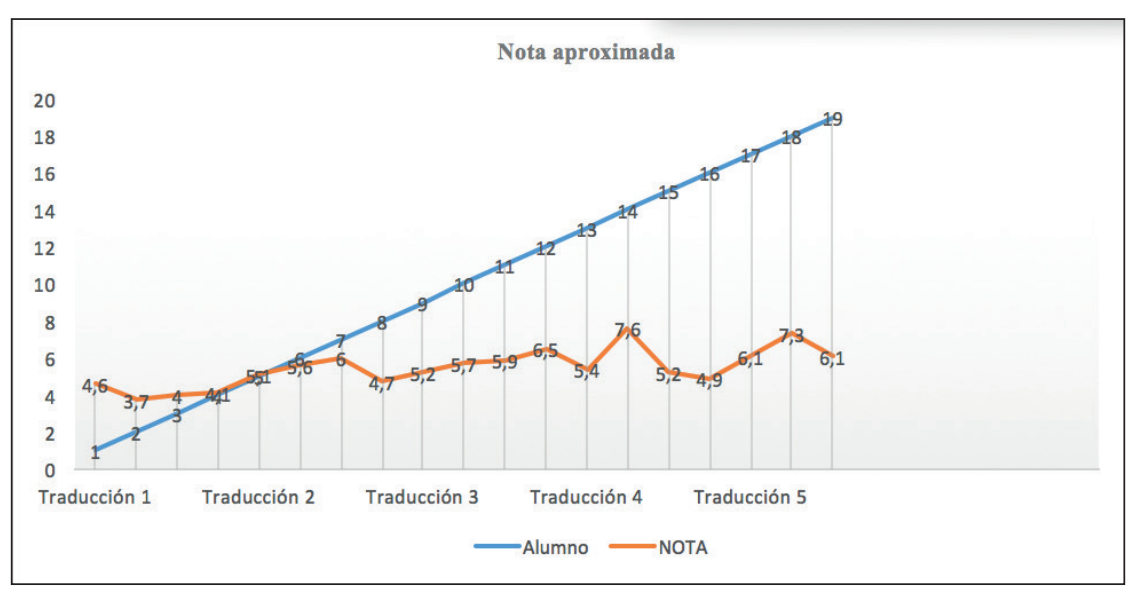

FIGURA 8.

Notas aproximadas de las traducciones en la asignatura Turismo II

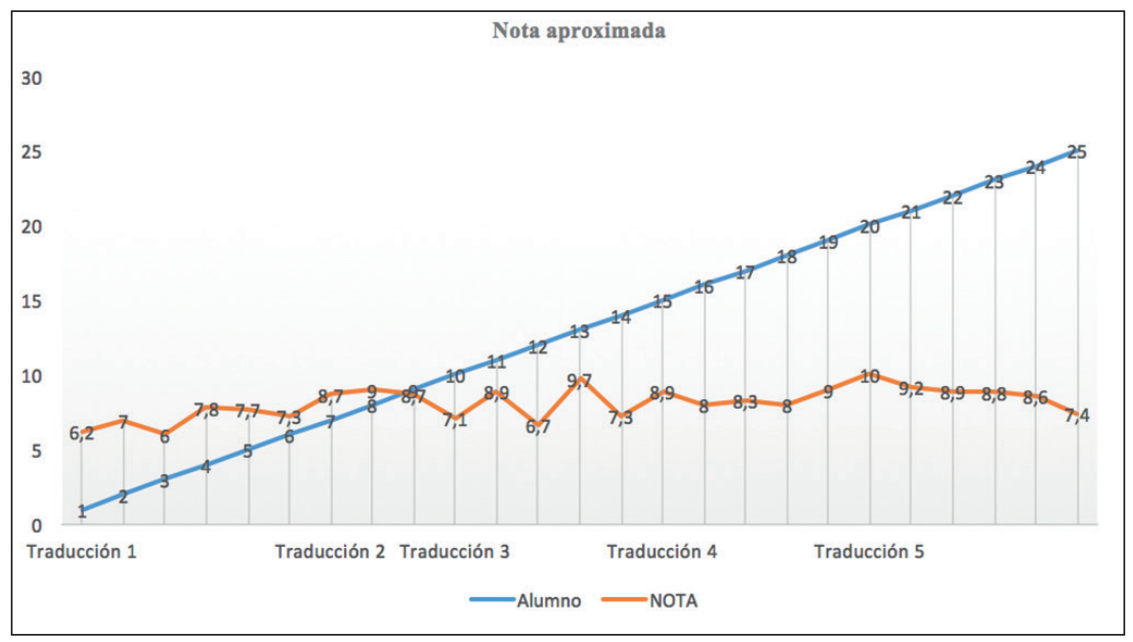


Figura 9.

Notas aproximadas de las traducciones en la asignatura Traducción Especializada

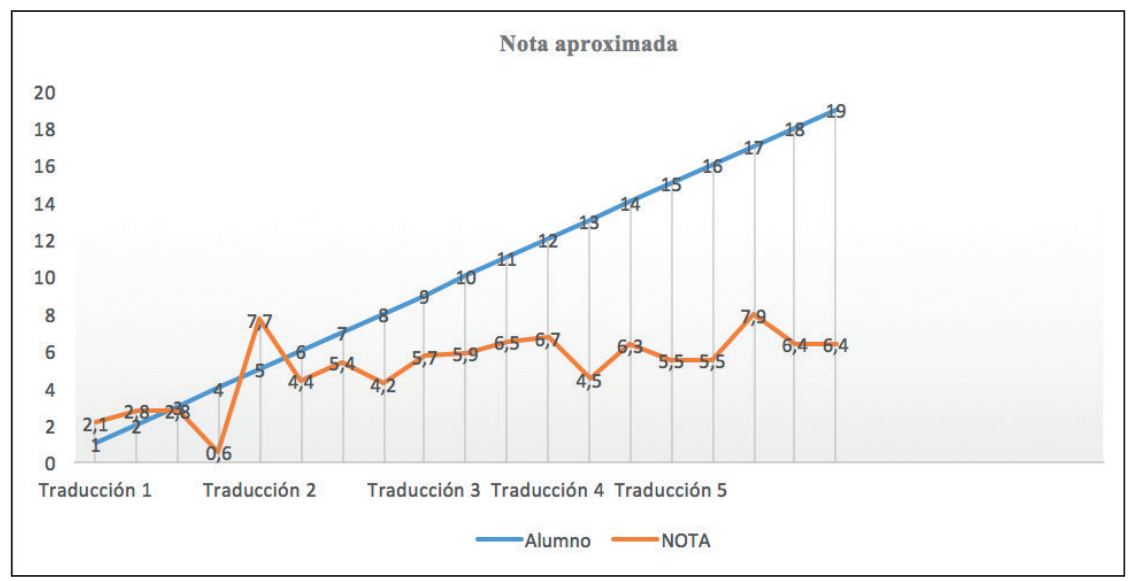

En cuanto a los resultados de la traducción del examen final, la media de todos los alumnos en las tres asignaturas arroja un resultado entre 9 y 10 . Todos estos aspectos van estrechamente relacionados con las subcompetencias expuestas en el marco teórico de este trabajo. La evaluación final de la asignatura muestra una radiografía de unos alumnos que tienen un conocimiento global porcentual entre el $90 \%$ y $100 \%$ de los conocimientos teórico-prácticos, con las siguientes subcompetencias:

- Bilingüe, incluyendo la competencia gramatical, la textual y la ilocucionaria.

- Extralingüística, incluyendo un conocimiento bicultural y temático.

— La relacionada con el conocimiento de la traducción.

- Instrumental, asociada al empleo de fuentes de documentación y tecnologías de la información.

—Estratégica que incluye a todas las anteriores.

\section{CONCLUSIÓN}

Tras el análisis detallado en torno a los datos arrojados en cada una de las fases del diseño del proyecto piloto (diagnóstico inicial, desarrollo de la asignatura y evaluación final de las competencias traductológicas y de la experiencia didáctica), puede afirmarse que los resultados obtenidos son los esperados en consonancia con la hipótesis de partida de este trabajo. Se prueba que la combinación de métodos e instrumentos en esta experiencia piloto es una buena base de partida para implantar la experiencia en otras asignaturas.

Sin embargo, cabe plantearse la posibilidad de un análisis de la calidad de las traducciones de forma externa centrándonos en un posible consumidor final del texto traducido. Esta evaluación externa nos aportaría una mayor cantidad de datos en torno al proyecto que vamos a proceder a implantar en otras asignaturas, facilitando la posible modificación de 
su diseño con parámetros no valorados hasta el momento. Recordemos que uno de los productos resultantes del proyecto piloto fue la creación de un blog por parte de los alumnos en cada una de las asignaturas. Este blog estaba compuesto por distintos blogs atendiendo al número de grupos de cada asignatura. Y cada uno de ellos estaba conformado por las distintas traducciones que los alumnos realizaron. Podrían establecerse dos evaluaciones externas en torno al blog. Una primera podría ser una coevaluación de las traducciones por grupos de clase distintos a los que realizaron las traducciones con las siguientes preguntas:

— ¿Atiende el uso del léxico al contenido principal?

— La sintaxis y distribución del texto, ¿está en consonancia con el contenido?

— ¿Transmite el léxico el mensaje?

- ¿Contribuyen los elementos sintácticos a transmitir el mensaje?

- ¿Apoya la actitud social el objetivo principal del texto?

— ¿Son los elementos léxicos apropiados para conseguir el objetico principal del texto?

— ¿Ayudan los elementos sintácticos y textuales a transmitir el mensaje?

— ¿Se adecúa el texto a la intención comunicativa?

- ¿Hay adecuación directa entre el texto y la intención comunicativa?

- ¿Hay relación entre el texto y el destinatario potencial?

- ¿Se utiliza la terminología de forma adecuada?

Otra evaluación externa podría ser una encuesta con las mismas preguntas enviadas a un público conocedor de las asignaturas pero externo al aula, como por ejemplo a otra clase de otra asignatura de la misma titulación. Podríamos valorar las respuestas de ambas encuestas con una escala psicométrica de Likert, con un rango del 1-5, donde 1 equivale a nada, y 5 a mucho.

\section{REFERENCIAS BIBLIOGRÁFICAS}

Bates, S., y Galloway, R. (2012). «The inverted classroom in a large enrolment introductory physics course: a case study». London: The Higher Education Academy STEM conference. Recuperado el 27 de enero de 2020 de

https://www2.ph.ed.ac.uk/ rgallowa/Bates_Galloway.pdf

Benítez, A. y M. García, (2013). «Un Primer Acercamiento al Docente frente a una Metodología Basada en Proyectos», Form. Univ., 6(1), 21-28

Bergmann, J., Y Sams, A. (2012). «Flip your classroom: Reach every student in every class every day». Eugene, Oregon, USA: International Society for Technology in Education (ISTE).

Bernard, R. M., Abrami, P. C., Borokhovski, E., Wade, C. A., Tamim, R. M., Surkes, M. A., Y Bethel, E. C. (2009). «A meta-analysis of three types of interaction treatments in distance education». Review of Educational Research, 79(3), 1243-1289. https://doi.org/10.3102/0034654309333844

Bernard, R. M., Borokhovski, E., Schmid, R. F., TAmim, R. M., y Abrami, P. C. (2014). «A meta-analysis of blended learning and technology use in higher education: From the general to the applied». Journal of Computing in Higher Education, 26(1), 87-122.

Blank, W. (1997). «Authentic instruction». En: W.E. Blank y S. Harwell (Eds.). Promising practices for connecting high school to the real world (pp. 15-21). Tampa, FL: University of South Florida. (ERIC Document Reproduction Service No. ED407586). 
Caraballo, M., Peinado, H., y González, M. (2017). «Gamificación en la educación, una aplicación práctica con la plataforma Kahoot». Anales de ASEPUMA, (25), 1-17. Recuperado el 27 de enero de $2020 \mathrm{de}$ https:// dialnet.unirioja.es/descarga/articulo/6210181.pdf

CieliebaK, M., y Frei, A. K. (2016). «Influence of flipped classroom on technical skills and non- technical competences of IT students». Paper presented at the Global Engineering Education Conference (EDUCON 2016). IEEExplore Digital Library. Recuperado el 27 de enero de 2020 de http://ieeexplore.ieee.org/document/7474676/

Crouch, C. H.; y Mazur, E. (2001). «Peer instruction: Ten years of experience and results». American Journal of Physics, 69, 9, September, pp. 970-977

Dickinson, K.P., Soukamneuth, S., Yu, H.c., Kimball, M., D’amico, R., Perry, R., et al. (1998). «Providing educational services in the Summer Youth Employment and Training Program [Technical assistance guide]». Washington, DC: U.S. Department of Labor, Ofice of Policy \& Research. (ERIC Document Reproduction Service No. ED420756).

Dziuban, C., Y Moskal, P. (2011). «A course is a course is a course: Factor invariance in student evaluation of online, blended and face-to-face learning environments». The Internet and Higher Education, 14(4), 236-241.

Dziuban, C., Hartman, J., Cavanagh, T., y Moskal, P. (2011). «Blended courses as drivers of institutional transformation». En A. Kitchenham (Ed.), Blended learning across disciplines: Models for implementation, (pp. 17-37). Hershey: IGI Global.

Elliot, R. (2014). «Do students like the flipped classroom? An investigation of student reaction to a flipped undergraduate IT course». Paper presented at the Frontiers in Education Conference (FIE 2014). IEEExplore Digital Library. Recuperado el 27 de enero de 2020 de http://ieeexplore.ieee.org/ document/7044070/

FERnÁndeZ-SÁnchez, N. (2011). «Promoción del cambio de estilos de aprendizaje y motivaciones en estudiantes de educación superior mediante actividades de trabajo colaborativo en blended learning». RIED. Revista Iberoamericana de Educación a Distancia, 14(2), 189-208. doi: https:// doi. org/10.5944/ried.2.14.795

Finkelstein, N., T. Hanson., C. W. Huang., B. Hirschman y M. Huang, (2010). «Effects of problem-based economics on high school economics instruction». U.S. Department of Education Washington, United States

FLORIDI, L. (2008). «A defence of informational structural realism». Synthese, 161(2), 219-253.

Flumerfelt, S., y Green, G. (2013). «Using Lean in the Flipped Classroom for At Risk Students». Educational Technology \& Society, 16(1), 356-366.

Fornons, V., y PALAU, R. F. (2016). «Flipped classroom en la asignatura de matemáticas de $3^{\circ}$ de Educación Secundaria Obligatoria». EDUTEC, Revista Electrónica de Tecnología Educativa, 55, 1-17. Recuperado el 27 de enero de 2020 de http://www.edutec.es/revista/index.php/edutec-e/article/ view/284

GARRISON, D. R., Y KANUKA, H. (2004). «Blended learning: Uncovering its transformative potential in higher education». The Internet and Higher Education, 7, 95-105.

GrahaM, C. R. (2013). «Emerging practice and research in blended learning». En M. G. Moore (Ed.), Handbook of distance education, (3rd ed., pp. 333-350). New York: Routledge.

Harwell, S. (1997). «Project-based learning». En: W.E. Blank y S. Harwell (Eds.), Promising practices for connecting high school to the real world (pp. 23-28). Tampa, FL: University of South Florida. (ERIC Document reproduction on Service No. ED407586).

Hurtado Albir, A. (1995). «La didáctica de la traducción. Evolución y estado actual», Perspectivas de la traducción (P. Hernandez y J. M. Bravo, dir.), Universidad de Valladolid, pp. 49-74.

Hurtado Albir, A. (2001). Traducción y Traductología. Madrid: Cátedra. 
Hurtado Albir, A. «The Acquisition of Translation Competence. Competences, Tasks, and Assessment in Translator Training». Meta 60/2 (2015): 256-280. Recuperado el 27 de enero de 2020 de

http://pagines.uab.cat/trec/content/hurtado-albir-“-acquisition-translation-competence-competences-tasks-and-assessment

JimÉnez, A., GÁmeZ, J., y Gómez, J. (2016). «Una propuesta para el refuerzo de conceptos matemáticos a través de Kahoot!». Revista del Congrés Internacional de Docència Universitària i Innovació (CIDUI). Recuperado el 27 de enero de 2020 de http://www.cidui.org/revistacidui/index.php/cidui/article/ view/1060/1024

Jonsson, H. (2015). «Using flipped classroom, peer discussion, and just-in-time teaching to increase learning in a programming course». Paper presented at the Frontiers in Education Conference (FIE 2015). IEEExplore Digital Library. Recuperado el 27 de enero de 2020 de http://ieeexplore.ieee. org/document/7344221/

Király, D. (1995): «Pathways to Translation», Pedagogy and Process, The Kent State University Press.

Lage, M. J.; Platt, G. J., Y Treglia, M. (2000). «Inverting the classroom: A gateway to creating an inclusive learning environment». The Journal of Economic Education, Vol. 31, $\mathrm{N}^{\circ}$ 1, Winter, pp. 30-43.

Margulieux, L. E., Mccracken, W. M., y Catrambone, R. (2016). «A taxonomy to define courses that mix face-to-face and online learning». Educational Research Review, 19, 104-118. doi: https://doi. org/10.1016/j.edurev.2016.07.001

Marlow, C. A. (2012). «The effect of the flipped classroom on student achievement and stress». Master of Science. Bozeman, Master's Thesis: Montana State University.

Means, B., Toyama, y., Murphy, R., Kaia, M., y Jones, K. (2010). «Evaluation of evidence-based practices in online learning». Washington: US Department of Education.

Means, B., Toyama, Y., MurPhy, R., y BaKi, M. (2013). «The effectiveness of online and blended learning: A meta-analysis of the empirical literature». Teachers College Record, 115(3), 1-47.

Molina, L. y Hurtado AlbiR, A. (2002). «Translation Techniques Revisited: A Dynamic and Functionalist Approach». Meta, 47 (4), 498-512. https://doi.org/10.7202/008033ar

Moreno, A., Ureña, J., Luna, M., De la Cruz, J., Roldán, T., Castro, T., Agugliaro, M., Salmerón, E., Gil, F., y Alcaide, A. (2018). «El uso de los sistemas de respuesta interactiva como herramienta para favorecer el aprendizaje proactivo en ingeniería». Revista de innovación y buenas prácticas docentes, 5, 91-96.

Neubert, A. (2000). «Competence in Language, in Languages, and in Translation». En C. Schäffner and B. Adab (eds.): Developing Translation Competence, Amsterdam, John Benjamins, pp. 3-18.

Norberg, A., Dziuban, C. D., y Moskal, P. D. (2011). «A time-based blended learning model». On the Horizon, 19(3), 207-216. https://doi.org/10.1108/10748121111163913

O'Flaherty, J., y Phillips, C. (2015). «The use of flipped classrooms in higher education: A scoping review». The Internet and Higher Education, 25, 85-95.

Ouadoud, M, Yassin Chkouri, M., NejJari, A. (2018). «LeaderTICE: A Platforms Recommendation System Based on a Comparative and Evaluative Study of Free E-learning Platforms». International Journal of Online and Biomedical Engineering, Vol. 14 (1), 132-161.

Olaizola, A. (2014). «La clase invertida: usar las TIC para "dar vuelta" a la clase». En Actas de las X Jornadas de Material Didáctico y Experiencias Innovadoras en Educación Superior, pp. 1-10, Buenos Aires.

PACTE (1998). «Procesos de aprendizaje y evaluación en la adquisición de la competencia traductora», European Society for Translation Studies, Granada Conference. 
Pacte (2000). «Acquiring Translation Competence: Hypotheses and Methodological Problems of a Research Project». En A. Beeby, D. Ensinger, M. Presas (ed.): Investigating Translation, Amsterdam, John Benjamins, pp. 99-106.

PACte (2003). «Building a Translation Competence Model». En Alves, F. (ed.): Triangulating Translation: Perspectives in Process Oriented Research, Amsterdam, John Benjamins, pp. 43-66.

PACTE (2005). «Investigating Translation Competence: Conceptual and Methodological Issues». Meta, 50 (2), pp. 609-618.

Perdomo, W. (2016). «Estudio de evidencias de aprendizaje significativo en un aula bajo el modelo flipped classroom». EDUTEC, Revista Electrónica de Tecnología Educativa, 55, 1-17. Recuperado el 27 de enero de 2020 de

http://www.edutec.es/revista/index.php/edutec-e/article/view/618/Edutec_n55_Perdomo

Picciano, A. G. (2009). «Blending with purpose: The multimodal model». Journal of Asynchronous Learning Networks, 13(1), 7-18.

Plump, C. M., Y Larosa, J. (2017). «Using Kahoot! in the Classroom to Create Engagement and Active Learning: A Game-Based Technology Solution for eLearning Novices». Management Teaching Review, 2(2), 151-158. https://doi.org/10.1177/2379298116689783

Ramos, S., Y Botella, A. (2017). «Innovación y Didáctica Musical para la docencia del siglo XXI en Educación Superior». DEDiCA. Revista de Educação e Humanidades (DREH), (12), 155-169. Recuperado el 27 de enero de 2020 de http://revistaseug.ugr.es/index.php/dedica/article/ view/6787/5905

RoAch, T. (2014). «Student perceptions toward flipped learning: New methods to increase interaction and active learning in economics». International Review of Economics Education, 17, 74-84.

Ross, B., y GAGE, K. (2006). «Global perspectives on blended learning: Insight from WebCT and our customers in higher education». En C. J. Bonk, \& C. R. Graham (Eds.), Handbook of blended learning: Global perspectives, local designs, (pp. 155-168). San Francisco: Pfeiffer.

Rovai, A. P., y Jordan, H. M. (2004). «Blended learning and sense of community: A comparative analysis with traditional and fully online graduate courses». International Review of Research. En Open and Distance Learning, 5(2), 1-13.

Sakai Learning Management System. (2019). Recuperado el 27 de enero de 2020 de https:// www.sakailms.org/

Saverv, J., (2006). «Overview of problem-based learning: Definitions and distinctions», 1(1), 9-20

Sife, A. S., Lwoga, E. ., Y SAnga, C. (2007). «New technologies for teaching and learning: Challenges for higher learning institutions in developing countries». International Journal of Education and Development Using Information and Communication Technology, Vol. 3(2), 57-67.

Sitzmann, T., Kraiger, K., Stewart, D., y Wisher, R. (2006). «The comparative effectiveness of web-based and classroom instruction: A meta-analysis». Personnel Psychology, 59(3), 623664.

Thomas, J.w., J.R. Mergendoller y A. Michaelson, (1999). «Project Based Learning: A handbook for middle and high school teachers», Novato, CA, The Buck institute for Education

Vega, F., E. Portillo, M. Cano y B. Navarrete, (2014). «Experiencias de aprendizaje en la ingeniería química: diseño, montaje y puesta en marcha de una unidad de destilación a escala de laboratorio mediante el aprendizaje basado en problemas». Form. Univ, 7(1), 13-22

Vizcarro, C.,V Juárez, E. (2008). «¿Qué es y cómo funciona el aprendizaje basado en problemas?», en El aprendizaje basado en problemas en la enseñanza universitaria, Universidad de Murcia, Servicio de Publicaciones, pp. 17-36 Murcia, España

Walvoord, B. Y Johnson, Anderson, V. (1998). Effective grading: A tool for learning and assessment. San Francisco: Jossey-Bass. 
Whiteside, A. L., Dikkers, A. G., Y Lewis, S. (2016). «More Confident Going into College: Lessons Learned from Multiple Stakeholders in a New Blended Learning Initiative». Online Learning, 20(4), 136- 156.

Willem, C., Aiello, M., Y Bartolomé, A. R. (2007). «Blended Learning and New Literacies». The International Journal of Technology, Knowledge \& Society, 2, 3-9.

Zhao, Y., Lei, J., Yan, B., LaI, C., Y TAN, H. S. (2005). «What makes the difference? A practical analysis of research on the effectiveness of distance education». Teachers College Record, 107(8), 1836-1884. https://doi.org/10.1111/j.1467-9620.2005.00544.x

Zhang, Y., Dang, Y., Y Amer, B. (2016). «A Large-Scale Blended and Flipped Class: Class Design and Investigation of Factors Influencing Students' Intention to Learn». IEEE Transactions on Education, 59(4), 263-273. [7435269]. https://doi.org/10.1109/TE.2016.2535205 
